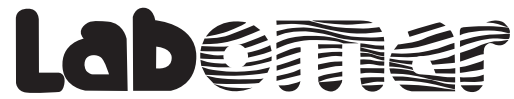

Arquivos de Ciências do Mar

\section{VARIAGÃO EM PEQUENA ESGALA DA MACROFAUNA BENTÔNICA EM UMA PLANÍCIE DE MARÉ DO ESTUÁRIO DO RIO PACOTI - CEARÁ, BRASIL}

\author{
Small-scale variation of the benthic macrofauna on a tidal \\ flat of Pacoti River estuary, Ceará, Brazil
}

\author{
Aline Ferreira da Silva1, Wilson Franklin-Júnior ${ }^{1}$, Cristina de Almeida Rocha-Barreira ${ }^{1 *}$ \\ ${ }^{1}$ Laboratório de Zoobentos do Instituto de Ciências do Mar, Universidade Federal do Ceará- LABOMAR-UFC, \\ Avenida Abolição, 3207, Meireles, Fortaleza-CE \\ *Autor para correspondência: cristina.labomar@gmail.com
}

\begin{abstract}
RESUMO
Em ambientes estuarinos, a interação entre as variações espaciais e temporais do ambiente controla a abundância e a diversidade dos organismos bentônicos. As feições do ambiente bentônico, bem como a variabilidade faunística, podem ser estudadas em diferentes escalas, sendo considerados fenômenos de pequena escala aqueles que caracterizam áreas de dezenas de $\mathrm{m}^{2}$ ou menos. O presente trabalho teve como objetivo avaliar a estrutura da macrofauna bentônica, correlacionando-a com as condições ambientais em pequena escala em uma planície de maré do estuário do rio Pacoti, Ceará. Uma área de 30×30 m sobre um banco areno-lamoso foi demarcada e subdividida em 9 subáreas de 10×10 m. A área apresentou alta similaridade faunística, verificando-se que as espécies se distribuíram em função das características do sedimento (a seleção do grão e os percentuais de carbonato de cálcio e de matéria orgânica), definindo assim a formação de agregações (manchas), ainda que pouco discriminadas, das espécies mais abundantes. As características espaciais em pequena escala representaram condições distintas que influenciaram na distribuição da macroinfauna, reforçando a íntima relação dos organismos bentônicos com os sedimentos e descrevendo um padrão de distribuição na área que, em avaliações horizontais de meso-escala, não seriam compreendidas.
\end{abstract}

Palavras-chave: banco areno-lamoso, distribuição espacial, Laeonereis acuta, Apseudidae.

\begin{abstract}
In estuarine environments, the interaction between spatial and temporal variations controls the abundance and diversity of benthic organisms. The features of the benthic environment, as well
\end{abstract}

Recebido: 12 dez 2016

Aceito: $1 .^{\circ}$ maio 2017

Publicado online: 31 mai 2017 
as the faunistic variability, can be studied in different scales, being considered small-scale phenomena those that characterize areas of tens of $m^{2}$ or less. The aim of the present research was to assess benthic macrofauna structure and correlate it to the environmental conditions in a tidal flat on Pacoti River estuary. In February of 2005, a tidal flat area of $30 \times 30 \mathrm{~m}$ was marked and subdivided in 9 areas of $10 \times 10 \mathrm{~m}$. The area presented high faunistic similarity, showing that species were distributed depending on the sediment characteristics (grain selection and the percentage of calcium carbonate and organic matter), thereby defining the formation of aggregates (spots) of the most abundant species, although they were weakly discriminated. Small-scale spatial features determined distinct conditions for the macroinfauna distribution, reinforcing the close relationship of benthic organisms to sediments and describing a pattern of distribution in the area that would not be understood in horizontal meso-scale assessments.

Keywords: sand-muddy bottom, spatial distribution, Laeonereis acuta, Apseudidae.

\section{INTRODUÇÃO}

A utilização da macrofauna bentônica para caracterizar as condições ambientais de ecossistemas costeiros é fundamental à ecologia, e entender o nível de relação dos seres vivos com os parâmetros ambientais (tipo de sedimento, $\mathrm{pH}$, teor de oxigênio dissolvido, temperatura, entre outros) no meio em que vivem é crucial em estudos de avaliação ambiental.

Por serem ecossistemas altamente produtivos e diversos, desempenharem papel fundamental na estabilidade da geomorfologia costeira, na conservação da biodiversidade, constituírem a base da cadeia trófica de espécies de importância ecológica (Monteiro, 2005), e proverem a manutenção de amplos recursos pesqueiros correspondendo, de alguma forma, a mais de $80 \%$ de captura marinha (Kjerfve et al., 1997), os manguezais merecem atenção redobrada no âmbito da pesquisa científica, principalmente no aspecto ecológico.

A maioria dos manguezais apresenta planícies flúvio-marinhas formadas pelo acúmulo de sedimentos graças ao baixo hidrodinamismo natural dessas áreas (Pinheiro, 2003; Lana et al., 1997). Neles, os processos estuarinos atuam como filtro à entrada de sedimentos (Couto $e t$ al., 1995). Estes ambientes recebem detritos provenientes do continente e do mar, além dos produzidos pela flora e fauna locais, que dão suporte a ricas associações bentônicas.

por distúrbios naturais e antropogênicos (Caswell \& Cohen, 1991). Em ambientes Padrões espaciais de distribuição de espécies são produzidos por uma interação entre variações ambientais, espacial e temporal, bem como por processos biológicos, e podem ser modificados estuarinos, esta variação controla espacial e temporalmente a abundância e a diversidade dos organismos bentônicos (Rosa-filho, 1997; Lana et al., 1997).

As variações espaciais da distribuição dos organismos nestas áreas podem ocorrer de centímetros a dezenas de metros, condicionadas por diversos fatores como diferentes condições hidrodinâmicas, estações do ano, distúrbios físicos episódicos, mudanças nas características sedimentares, migração, recrutamento, competição, predação e produtividade (Schaffner, 1990; Morrissey et al., 1992). Em vista disto, os padrões de dispersão da fauna bentônica podem ser mais evidentes em certas escalas e pouco perceptíveis em outras, sendo estes pouco estudados, particularmente em regiões tropicais e subtropicais. De acordo com Zajac (1999), as feições do ambiente bentônico, bem como a variabilidade fau- 
nística, podem ser estudadas em diferentes escalas, sendo considerados fenômenos de pequena escala aqueles que caracterizam áreas de dezenas de $\mathrm{m}^{2}$ ou menos.

No estado do Ceará, os campos de mangues se apresentam em toda a extensão de seu litoral, associados a estuários e lagunas (Monteiro, 2005). O estuário do rio Pacoti localiza-se no litoral leste do Estado, estando inserido na bacia inferior do rio Pacoti, a qual abrange uma área de cerca de 700 km² nos municípios de Aquiraz, Pacatuba e Euzébio (Lacerda \& Sena, 2005). Este rio é um dos principais representantes dos recursos hídricos do Ceará e o maior dos cursos d'água que atravessam a região metropolitana de Fortaleza.

Tendo em vista as considerações feitas acima, o presente trabalho teve como objetivo avaliar as variações em pequena escala da estrutura da comunidade da macroinfauna bentônica em uma planície de maré no estuário do rio Pacoti, Ceará - Brasil, correlacionando a composição específica, abundância, diversidade, riqueza e equitabilidade com as variações espaciais em pequena escala dos parâmetros ambientais, temperatura, salinidade e oxigênio dissolvido na água de percolação, e carbonato de cálcio, matéria orgânica e características granulométricas dos sedimentos.

\section{MATERIAL E MÉTODOS}

Para a realização do estudo, foi demarcada, sobre uma planície de maré situada na margem esquerda da foz do rio Pacoti $\left(03^{\circ} 49^{\prime} 13^{\prime \prime}\right.$; $038^{\circ} 24^{\prime} 8^{\prime \prime} \mathrm{W}$ ) (Figura 1), uma área de 30x30 m, dividida em 9 subáreas de 10x10 m. De cada subárea, foram retiradas cinco amostras de sedimento para análise biológica, totalizando 45 amostras. A numeração das subáreas foi iniciada pela extremidade mais interna (em direção ao rio) e próxima ao bosque de mangue, seguindo-se as demais subáreas em linhas horizontais (Figura 2b).

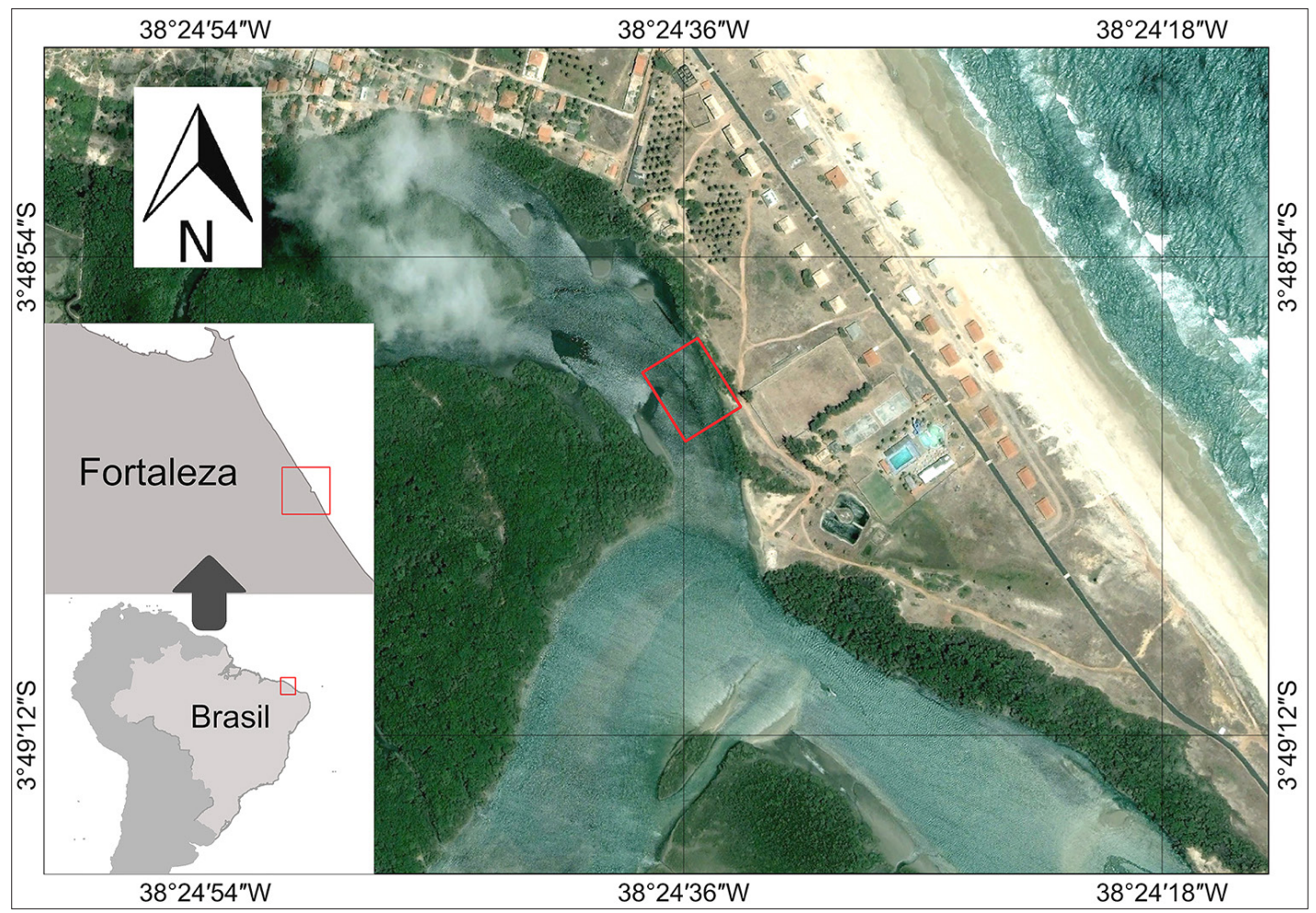

Figura 1 - Localização do banco areno-lamoso estudado no estuário do Rio Pacoti, Município de Eusébio, Ceará. Fonte: Adaptado de Google Earth. 
As amostras foram obtidas com o uso de um amostrador cilíndrico de PVC com $15 \mathrm{~cm}$ de diâmetro, o qual foi enterrado a $10 \mathrm{~cm}$ de profundidade no sedimento (Franklin-Júnior, 2000). As amostras foram armazenadas em sacos plásticos, etiquetadas, preservadas em solução salina de formaldeído a 4\%. Para análise granulométrica, foi coletada uma amostra de cada subárea, utilizando o mesmo amostrador das amostras biológicas. O teor de oxigênio, salinidade e temperatura da água de percolação do banco areno-lamoso foram mensurados no momento da coleta.

Em laboratório, as amostras foram peneiradas em uma malha de 0,5 mm de abertura, lavadas com água corrente e armazenadas em álcool $70 \%$ com o corante Rosa de Bengala. Os organismos foram triados, separados em grupos taxonômicos e, posteriormente, identificados até o menor nível taxonômico possível. A triagem e identificação dos organismos foram realizadas utilizando-se estereomicroscópio, com aumento de até 40x, e microscópio óptico com aumento de

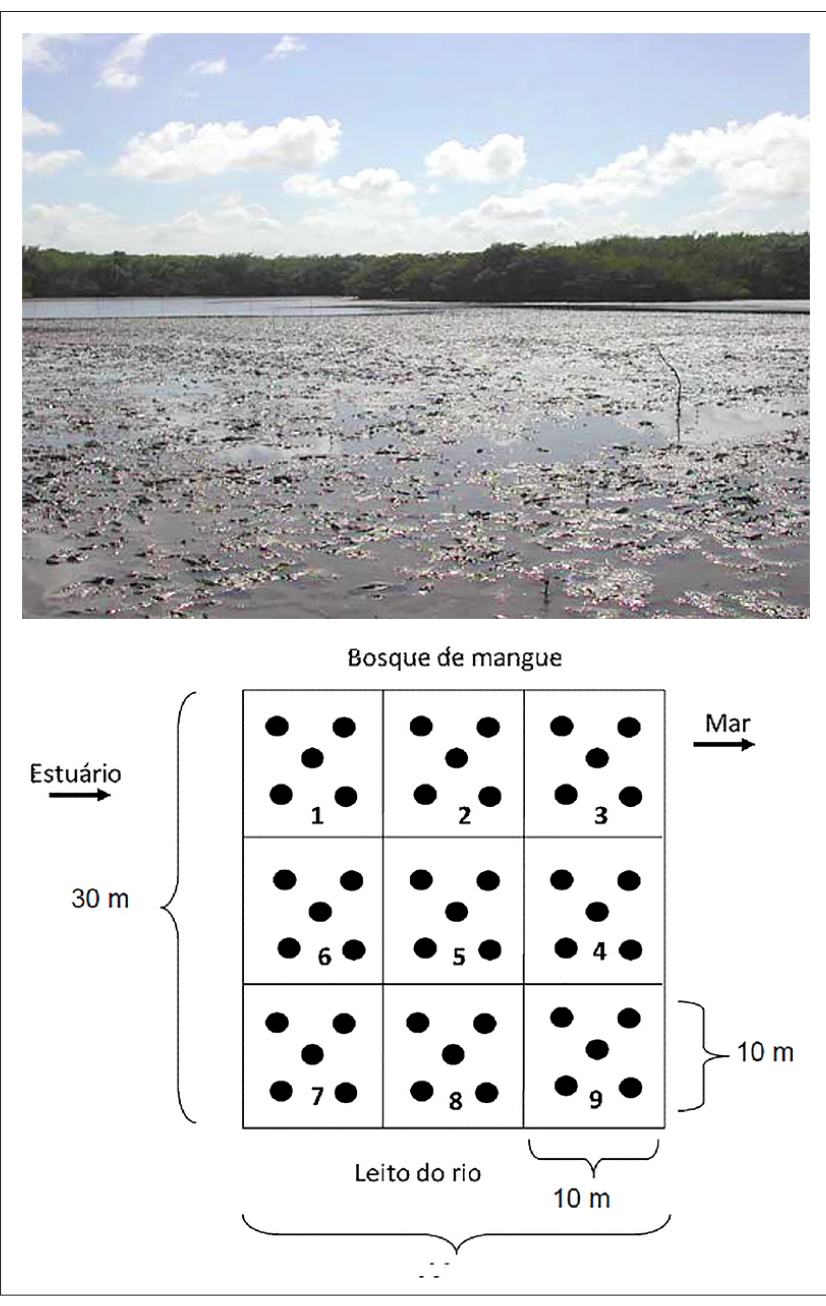

Figura 2 - (a) Banco areno-lamoso no estuário do rio Pacoti exposto durante a maré baixa, Eusébio, Ceará; (b) Desenho esquemático da estratégia amostral empregada neste estudo. até $1000 x$.

As análises granulométricas do sedimento foram realizadas no Laboratório de Geologia Marinha do Instituto de Ciências do Mar da Universidade Federal do Ceará. As frações de sedimento grosseiro (areias) foram quantificadas através do método de peneiramento a seco e as frações de sedimento fino (silte e argila) através do método de pipetagem. Os parâmetros estatísticos, média, desvio padrão, seleção, assimetria, curtose e classificação textural foram obtidos, através do programa SYSGRAN ${ }^{\circledR}$ versão 2.0.

Os teores de carbonato de cálcio foram determinados através do método do Calcímetro de Bernard modificado. A matéria orgânica presente no sedimento foi determinada por técnica gravimétrica que consiste na queima de $2 \mathrm{~g}$ do sedimento por $2 \mathrm{~h}$ em mufla a $450^{\circ} \mathrm{C}$. O teor de matéria orgânica foi determinado pela diferença de peso da amostra antes e depois da queima.

A macrofauna bentônica foi caracterizada através da determinação dos índices de Diversidade de Shannon-Wiener ( $\mathrm{H}^{\prime}$ pelo $\log _{\mathrm{e}}$ ), Riqueza (D de Margalef) e Equitabilidade de Pielou (J') a partir dos valores de abundância média das espécies, considerando as 5 réplicas analisadas para cada ponto amostrado.

O grau de associação entre a macrofauna bentônica presente nos pontos de coleta (análise normal - modo Q) foi determinado, com base na abundância média por ponto, 
através da análise de agrupamento do tipo hierárquico aglomerativo utilizando-se o coeficiente de Bray-Curtis e como regra de união a média não ponderada (UPGMA). Para reduzir as discrepâncias entre os dados analisados, as matrizes originais sofreram transformação prévia do tipo $\mathrm{y}=\log (\mathrm{x}+1)$. As matrizes de similaridade foram calculadas e submetidas à ordenação por escalonamento multidimensional (MDS). Foi realizada, ainda, a análise SIMPER (Clarke \& Warwick, 1994) com a finalidade de identificar as espécies que tiveram maior contribuição para a formação dos grupos observados nas análises de cluster e no MDS.

Foram examinadas as relações entre os descritores da estrutura da comunidade e as variáveis ambientais através do procedimento BIOENV (Clarke \& Warwick, 1994). Para subsidiar a seleção dos descritores ambientais a serem utilizadas na análise BIOENV, utilizou-se a rotina DRAFTSMAN PLOT entre todas as variáveis, visando determinar eventuais colinearidades (rs>0,95), retirando das análises os parâmetros variáveis altamente correlacionados.

Foi calculado o índice de dispersão de Morisita (1962) para indicar o padrão de distribuição dos táxons identificados, utilizando- se a seguinte equação:

$$
I_{\delta}=n \frac{\Sigma[x(x-1)]}{\Sigma x(\Sigma x-1)}=n \frac{\Sigma x^{2}-\Sigma x}{(\Sigma x)^{2}-\Sigma x}
$$

onde: $\mathrm{n}=$ número de unidades amostrais e $\sum \mathrm{x}=$ somatória do número de indivíduos presentes nos quadrados amostrados. A distribuição será aleatória quando Id $=1$, agregada quando Id $>1$ e uniforme quando $\mathrm{Id}<1$. O afastamento da aleatoriedade pode ser testado por:

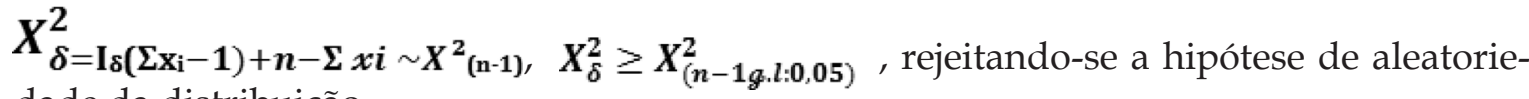
dade da distribuição.

As análises de variância e correlação foram realizadas utilizando o programa STATISTICA for windows ${ }^{\circledR}$ versão 6.0. A determinação dos descritores da comunidade, bem como, as análises de agrupamento, MDS, Simper e BIOENV foram realizadas utilizando o programa PRIMER (Plymouth Routines In Multivariate Ecological Research) for Windows versão 5.2.4. O cálculo do índice de Morisita foi realizado em planilha do Microsoft Excel ${ }^{\circledR}$ versão 2013.

\section{RESULTADOS}

\section{Descritores abióticos da planície de maré do estuário do rio Pacoti}

O sedimento caracterizou-se predominantemente por areia fina e percentuais consideráveis de silte e argila, o que caracteriza esta planície de maré como um banco areno-lamoso (Tabela 1). A única exceção foi a subárea 1, na parte mais superior do banco e próximo ao bosque de mangue, que foi constituído por areia média. A área apresentou também uma pequena parcela de cascalho composto por fragmentos de conchas vazias de moluscos bivalves e restos de troncos da vegetação de mangue (Tabela 2).

A subárea 1 também caracterizou-se pela menor porcentagem de cascalho e a subárea 6, na região lateral do banco mais interna (em direção ao rio), com maior valor. Nesses locais, subáreas 1 e 6, a porcentagem do teor de carbonato de cálcio apresentou valor mínimo e máximo de $0,67 \%$ e 14,14\% respectivamente. O diâmetro médio dos grãos variou de 1,82 $\Phi$ na subárea 1 a 2,67 $\Phi$ na subárea 5, na região central do banco. O maior 
percentual de areia foi na subárea 1 com 91,61\% e o menor percentual na subárea 2 com $73,20 \%$. Nesses locais também se observou a maior variação da quantidade de finos, que passou de $8,31 \%$ na subárea 1 para $26,58 \%$ na subárea 2 .

Os valores registrados para o teor de matéria orgânica das subáreas amostradas apontaram a menor porcentagem na subárea 1 com 2,29\% e maior na subárea 2 com $6,87 \%$ (Tabela 2).

Os valores de oxigênio dissolvido foram baixos. A subárea 6 foi a única com valor de 1,23 mg/L e as demais subáreas apresentaram concentrações abaixo deste, sendo a subárea 8, na margem inferior próximo ao leito do rio, a de menor concentração do gás, com $0,16 \mathrm{mg} / \mathrm{L}$ (Tabela 2). A temperatura registrada no sedimento apresentou valores relativamente uniformes, variando de $31,7^{\circ} \mathrm{C}$ a $34,0^{\circ} \mathrm{C}$. A salinidade foi mais elevada nas subáreas 2 e 3, mais próximas ao manguezal, com 40 e o menor valor foi retratado na subárea 6 com 36 (Tabela 2).

Tabela 1 - Características granulométricas e classificação do sedimento do banco areno-lamoso do estuário do rio Pacoti (CE) observadas durante o período de estudo.

\begin{tabular}{cccccccc}
\hline Ponto & $\begin{array}{c}\text { Média do } \\
\text { grão }(\Phi)\end{array}$ & $\begin{array}{c}\text { Desvio } \\
\text { Padrão }\end{array}$ & $\begin{array}{c}\text { Cascalho } \\
(\%)\end{array}$ & Areia $(\%)$ & Silte $(\%)$ & Argila (\%) & Classificação \\
\hline 1 & 1,82 & 0,60 & 0,09 & 91,61 & 7,51 & 0,80 & Areia Média \\
2 & 2,60 & 0,72 & 0,22 & 73,20 & 25,69 & 0,89 & Areia Fina \\
3 & 2,60 & 0,74 & 0,13 & 75,90 & 21,97 & 1,99 & Areia Fina \\
4 & 2,47 & 0,69 & 0,38 & 80,92 & 16,31 & 2,39 & Areia Fina \\
5 & 2,67 & 0,65 & 0,35 & 73,60 & 22,26 & 3,79 & Areia Fina \\
6 & 2,54 & 0,64 & 0,82 & 77,52 & 19,53 & 2,13 & Areia Fina \\
7 & 2,38 & 0,64 & 0,79 & 82,62 & 14,94 & 0,79 & Areia Fina \\
8 & 2,31 & 0,72 & 0,42 & 83,95 & 13,60 & 2,03 & Areia Fina \\
9 & 2,37 & 0,69 & 0,22 & 83,71 & 13,35 & 0,79 & Areia Fina \\
\hline
\end{tabular}

Tabela 2 - Parâmetros abióticos do sedimento do banco areno-lamoso do estuário do rio Pacoti (CE) medidos durante o período de estudo.

\begin{tabular}{cccccc}
\hline Ponto & $\begin{array}{c}\mathrm{CaCO} 3 \\
(\%)\end{array}$ & $\begin{array}{c}\text { Matéria } \\
\text { orgânica }(\%)\end{array}$ & Salinidade & $\begin{array}{c}\text { Oxigênio } \\
(\mathrm{mg} / \mathrm{L})\end{array}$ & $\begin{array}{c}\text { Temperatura } \\
\left({ }^{\circ} \mathrm{C}\right)\end{array}$ \\
\hline 1 & 0,67 & 2,29 & 37 & 0,50 & 34,0 \\
2 & 6,73 & 6,87 & 40 & 0,26 & 33,3 \\
3 & 4,71 & 4,15 & 40 & 0,31 & 33,6 \\
4 & 3,37 & 3,23 & 38 & 0,31 & 31,8 \\
5 & 6,73 & 5,02 & 39 & 0,22 & 31,7 \\
6 & 14,14 & 3,12 & 36 & 1,23 & 32,0 \\
7 & 3,37 & 2,87 & 38 & 0,76 & 32,0 \\
8 & 4,04 & 2,44 & 39 & 0,16 & 33,5 \\
9 & 6,40 & 2,55 & 39 & 0,22 & 34,0 \\
\hline
\end{tabular}

\section{Descritores bióticos da planície de maré do estuário do rio Pacoti}

Foram identificados 12.125 indivíduos, pertencentes a 31 táxons da macrofauna bentônica e distribuídos em 15 espécies de poliquetas, 8 espécies de moluscos e 8 grupos de crustáceos.

Considerando o total de organismos encontrados, as espécies Laeonereis culveri com $68 \%$ e Capitella spp. com 14\% apresentaram maior abundância média em todos os pontos amostrados, com densidades de 10.423 ind. $/ \mathrm{m}^{2}$ e 2.141 ind./ $\mathrm{m}^{2}$, respectivamente. Em se- 
guida, o crustáceo Apseudidae representou 10\% do total de indivíduos com densidade de $1.641 \mathrm{ind} / \mathrm{m}^{2}$, superando valores de abundância média dos poliquetas e moluscos apenas na subárea 4, na margem próxima à água voltada para o mar. Com valores inferiores a 1\%, os moluscos ocorreram em menor frequência ao longo dos 9 pontos amostrados, Tagelus plebeius se sobressaiu apresentando uma densidade de 81 ind. $/ \mathrm{m}^{2}$.

A espécie Laeonereis culveri representou $77 \%$ do total das espécies de poliquetas, seguida de crustáceos Apseudidae com 99\% do total de indivíduos do grupo e, por fim, o bivalve Tagelus plebeius com $42 \%$ do número total de moluscos.

Considerando o padrão de dispersão das espécies sobre o banco areno-lamoso, em virtude de um grande número de espécies pouco abundantes, verificou-se um predomínio do padrão agregado. Entretanto, entre as espécies mais abundantes, os poliquetas Laeonereis culveri $(\mathrm{Id}=0,9 ; \mathrm{p}<0,05)$ e Heteromastus similis $(\mathrm{Id}=1,2 ; \mathrm{p}<0,05)$ apresentaram um padrão de dispersão aleatório, o bivalve Tagelus plebeius apresentou padrão uniforme $(\operatorname{Id}=0,8 ; p>0,05)$ e os crustáceos Apseudidae apresentaram um padrão agregado ( $I d=3,0 ; p<0,05)$ (Tabela 3 ).

Foi observada uma baixa eqüitabilidade ao longo da área, com valor mais elevado na subárea 4 e menos elevado na subárea 6. Ainda na subárea 4, ocorreram os maiores índices de diversidade e abundância e os menores valores desses descritores foram registrados nas subáreas 6 e 2 respectivamente. Quanto à riqueza, o maior índice foi na subárea 3 e o menor na subárea 1.

A partir do somatório dos valores médios, considerando cada subárea de coleta, a estrutura da comunidade quanto aos grupos taxonômicos (poliquetas, moluscos e crustáceos), revelou os poliquetas como representantes mais expressivos de todo o plano intermareal. Nas subáreas 1 e 2, mais próximas ao bosque de mangue, estes organismos representaram os maiores valores percentuais com $99 \%$ de representatividade. Os moluscos contribuíram com valor máximo de apenas $2 \%$ na subárea 5 , na região central do plano, e os crustáceos apresentaram o percentual mais elevado na subárea 4, na margem próxima à água voltada para o mar, com 43\% (Figura 3).

Tabela 3 - Análise quantitativa da macrofauna bentônica do banco areno-lamoso do estuário do rio Pacoti. Valores em negrito representam a abundância média e entre parênteses o desvio padrão.

\begin{tabular}{|c|c|c|c|c|c|c|c|c|c|c|c|c|}
\hline Espécies / Pontos & 1 & 2 & 3 & 4 & 5 & 6 & 7 & 8 & 9 & ind $/ \mathrm{m}^{2}$ & $\%$ ind $*$ & $\%$ ind $^{* *}$ \\
\hline \multicolumn{13}{|l|}{ Poliquetas } \\
\hline \multirow[t]{2}{*}{ Laeonereis culveri } & 227,0 & 167,8 & 195,4 & 133,8 & 212,8 & 208,4 & 176,2 & 160,4 & 169,2 & 10422,98 & 77,45 & 68,04 \\
\hline & $(70,2)$ & $(69,6)$ & $(61,5)$ & $(57,2)$ & $(83,2)$ & $(51,5)$ & $(68,9)$ & $(38,0)$ & $(31,0)$ & & & \\
\hline \multirow[t]{2}{*}{ Capitella spp. } & 52,6 & 34,8 & 54,8 & 35,8 & 37,2 & 20,8 & 42,0 & 34,8 & 26,4 & 2141,41 & 15,91 & 13,98 \\
\hline & $(17,8)$ & $(23,6)$ & $(25,1)$ & $(19,0)$ & $(27,9)$ & $(20,4)$ & $(32,5)$ & $(28,5)$ & $(24,7)$ & & & \\
\hline \multirow[t]{2}{*}{ Heteromastus similis } & 5,2 & 2,0 & 12,4 & 21,8 & 7,2 & 4,2 & 5,6 & 9,8 & 8,4 & 483,59 & 3,59 & 3,16 \\
\hline & $(6,1)$ & $(1,6)$ & $(5,4)$ & $(8,6)$ & $(8,0)$ & $(4,0)$ & $(4,0)$ & $(5,5)$ & $(6,9)$ & & & \\
\hline \multirow[t]{2}{*}{ Sigambra grubii } & 12,4 & 4,8 & 5,6 & 5,0 & 4,8 & 3,6 & 5,6 & 2,4 & 4,0 & 304,29 & 2,26 & 1,99 \\
\hline & $(3,6)$ & $(6,1)$ & $(5,9)$ & $(2,0)$ & $(6,5)$ & $(3,0)$ & $(3,9)$ & $(2,7)$ & $(4,2)$ & & & \\
\hline \multirow[t]{2}{*}{ Streblospio cf. benedict } & 3,0 & 0,8 & 1,8 & 0,0 & 1,2 & 0,8 & 1,6 & 0,4 & 0,0 & 60,61 & 0,45 & 0,40 \\
\hline & $(2,2)$ & $(0,8)$ & $(3,0)$ & $(0,0)$ & $(1,3)$ & $(1,3)$ & $(2,3)$ & $(0,5)$ & $(0,0)$ & & & \\
\hline \multirow[t]{2}{*}{ Glycinde multidens } & 0,0 & 0,0 & 1,2 & 0,2 & 0,2 & 0,0 & 0,6 & 0,0 & 0,4 & 16,41 & 0,12 & 0,11 \\
\hline & $(0,0)$ & $(0,0)$ & $(0,8)$ & $(0,4)$ & $(0,4)$ & $(0,0)$ & $(0,9)$ & $(0,0)$ & $(0,5)$ & & & \\
\hline \multirow[t]{2}{*}{ Marphysa formosa } & 0,0 & 0,0 & 0,0 & 0,6 & 0,4 & 0,2 & 0,4 & 0,2 & 0,0 & 11,36 & 0,08 & 0,07 \\
\hline & $(0,0)$ & $(0,0)$ & $(0,0)$ & $(0,5)$ & $(0,5)$ & $(0,4)$ & $(0,5)$ & $(0,4)$ & $(0,0)$ & & & \\
\hline \multirow[t]{2}{*}{ Typosyllis sp. } & 0,0 & 0,0 & 0,4 & 0,0 & 0,2 & 0,0 & 0,0 & 0,2 & 0,0 & 5,05 & 0,04 & 0,03 \\
\hline & $(0,0)$ & $(0,0)$ & $(0,5)$ & $(0,0)$ & $(0,4)$ & $(0,0)$ & $(0,0)$ & $(0,4)$ & $(0,0)$ & & & \\
\hline \multirow[t]{2}{*}{ Typosyllis hyalina } & 0,0 & 0,2 & 0,2 & 0,0 & 0,0 & 0,0 & 0,0 & 0,0 & 0,0 & 2,53 & 0,02 & 0,02 \\
\hline & $(0,0)$ & $(0,4)$ & $(0,4)$ & $(0,0)$ & $(0,0)$ & $(0,0)$ & $(0,0)$ & $(0,0)$ & $(0,0)$ & & & \\
\hline
\end{tabular}


(continuação Tabela 3)

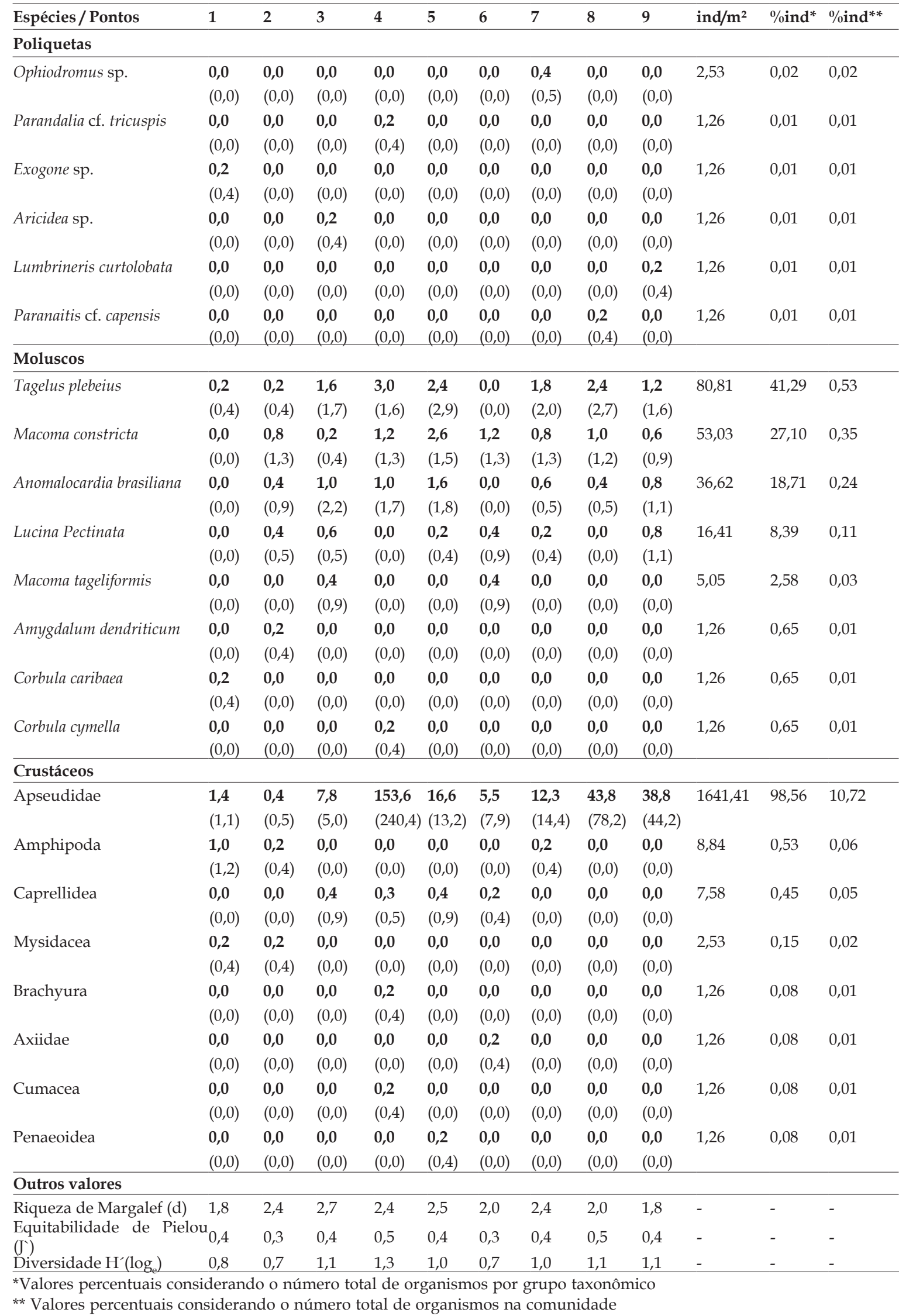




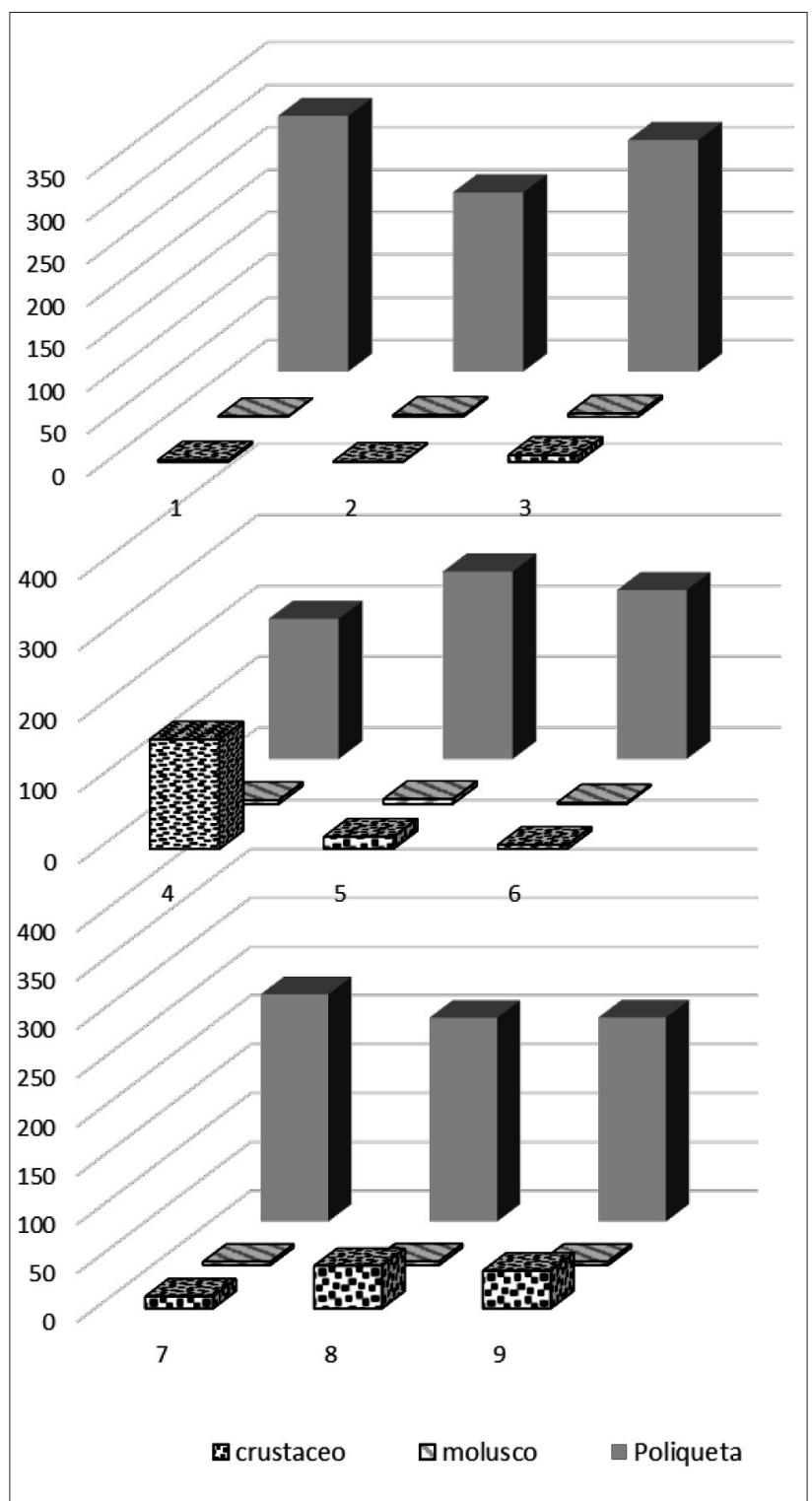

Figura 3 - Abundância relativa de crustáceos, moluscos e poliquetas observados ao longo das subáreas de coleta (1 a 9) no banco areno-lamoso do rio Pacoti - CE.

\section{Análise de agrupamento da macrofauna bentônica no banco areno-lamoso do estuário do rio Pacoti}

Através da análise de agrupamento do rio Pacoti, foi possível observar a formação de 2 grupos bem definidos. O grupo 1 apresentou semelhança superior a $80 \%$ e reuniu a maior parte das subáreas (67\% de todos as subáreas) e o grupo 2 que teve $85 \%$ de semelhança e foi constituído pelas subáreas 1, 2 e 6 (Figura 4). Vale ressaltar que os resultados do Cluster e MDS mostraram os mesmos grupos nas duas análises (Figura 5).

De acordo com a análise de SIMPER, foi possível reconhecer as principais espécies e sua contribuição para a formação dos grupos. O grupo 1a com $87 \%$ de similaridade apresentou como espécies mais representativas os poliquetas Laeonereis culveri com $74 \%$ de participação, e Capitella spp. com 16\%, seguido do crustáceo Apseudidae com apenas 4\% de participação para a formação do grupo. 


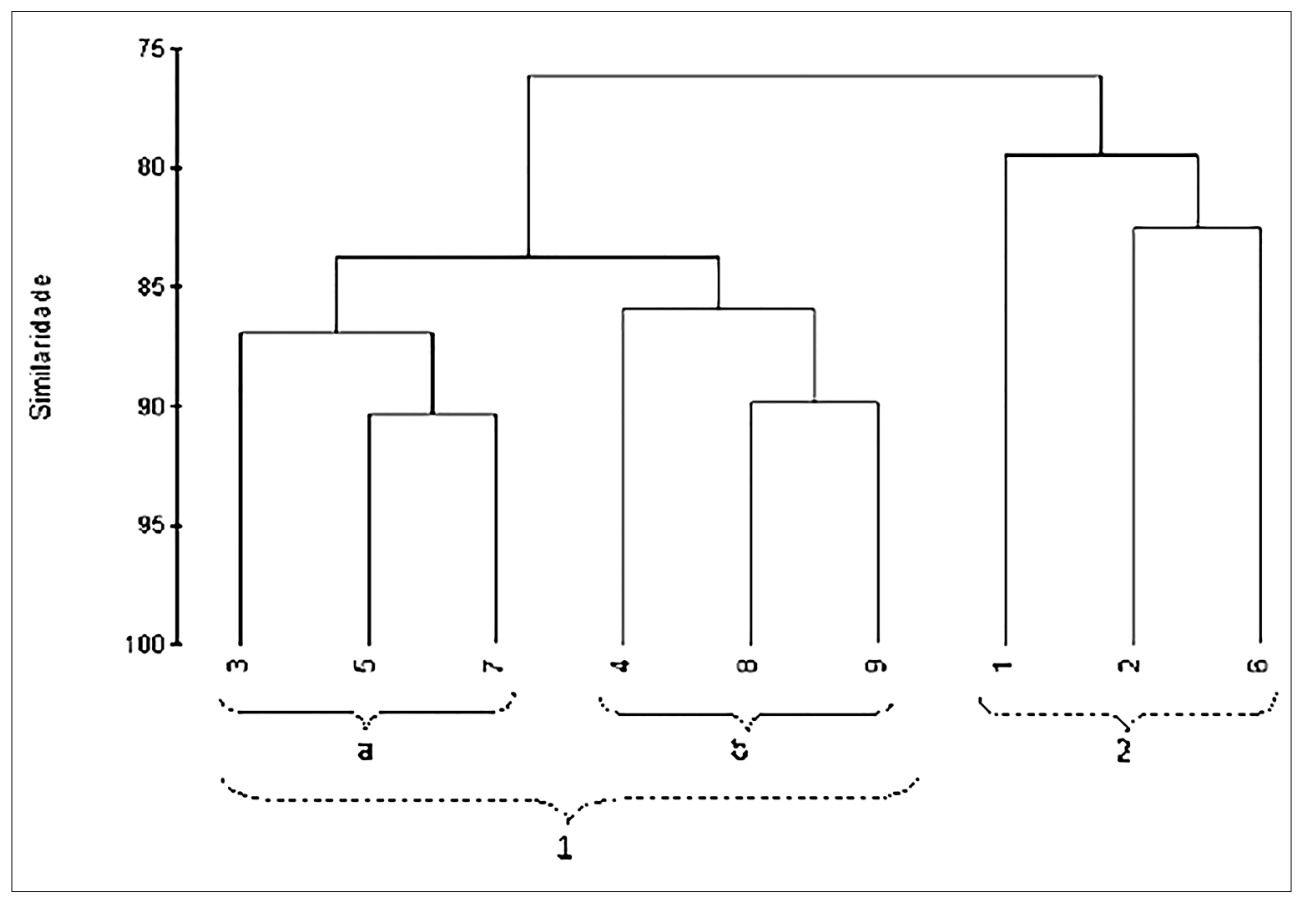

Figura 4 - Resultado da análise de agrupamento utilizando a distância de Bray Curtis, pelo método UPGMA, com as subáreas amostradas do banco areno-lamoso do rio Pacoti - CE.

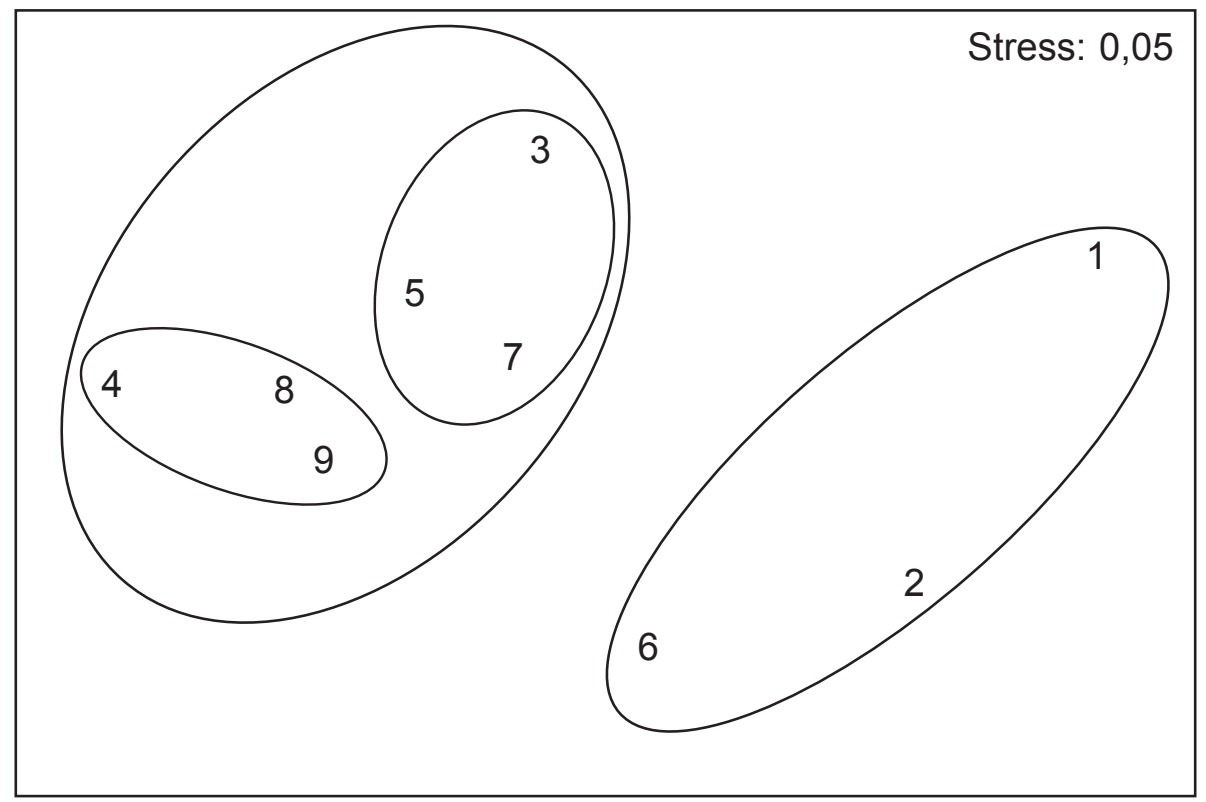

Figura 5 - Ordenação MDS das subáreas de acordo com a macrofauna bentônica no banco areno-lamoso do estuário do rio Pacoti - CE obtida dos dados de abundância média das espécies coletadas.

O grupo 1b, com 86\% de similaridade, reuniu subáreas com baixos teores de oxigênio dissolvido e maiores valores de temperatura, sendo possível verificar pontos com elevados valores de abundância média, equitabilidade e diversidade. A análise de SIMPER discriminou as espécies L. culveri com 63\%, crustáceos Apseudidae com 18\% e Capitella spp. com $13 \%$ de contribuição para a formação do grupo. 
O grupo 2 apresentou $80 \%$ de semelhança e foi constituído pelas subáreas 1, 2 e 6 . Este grupo caracterizou-se por uma área de sedimento de areia média a fina, valores altos de cascalho, carbonato de cálcio, finos e matéria orgânica, salinidade, oxigênio e temperatura, e ainda, menor riqueza e diversidade. Nessa área, através da análise de SIMPER, foi possível reconhecer a ocorrência principalmente das espécies Laeonereis culveri com $84 \%$ e Capitella spp. com $12 \%$ de contribuição, podendo citar ainda espécies como Parandalia cf. tricuspis, Lumbrineris curtolobata e Paranaitis cf. capensis que ocorreram uma única vez em subáreas que constituíram o grupo 2.

A análise de BIOENV discriminou a seleção do grão, o teor de carbonato de cálcio e teor de matéria orgânica como os fatores abióticos que melhor explicaram a estrutura da macrofauna bentônica do banco areno-lamoso do rio Pacoti $(r=0,571)$.

\section{DISCUSSÃO}

De acordo com Oliveira et al. (1988), o caráter granulométrico de um estuário representa um fator essencial na estruturação da comunidade bêntica local. Nanami et al. (2005) demonstraram uma clara diferença espacial na estrutura das assembleias macrobênticas em relação às variáveis ambientais, em particular, tamanho médio do grão e salinidade de forma independente.

O banco de lama do estuário do Pacoti apresentou um sedimento pouco selecionado, porém com elevada densidade da macrofauna bentônica. A riqueza específica da macrofauna apresentou uma forte correlação com o tamanho médio do grão e com os percentuais de silte, areia e matéria orgânica. Tal resultado foi reforçado quando, através do BIOENV, foram discriminados a seleção do grão e os percentuais de carbonato de cálcio e de matéria orgânica como os fatores abióticos mais importantes na estruturação da comunidade macrobentônica. Lana et al. (1997) registraram, na baia de Paranaguá, que os menores valores de riqueza e abundância foram nos setores mesoalinos, caracterizados por sedimentos finos e elevados conteúdos de carbono orgânico.

Desta forma, pode-se observar que o percentual de matéria orgânica foi o fator destacado nas análises do presente estudo. A maior concentração de matéria orgânica $(6,88 \%)$ ocorreu em áreas que havia maior ocorrência de sedimentos finos, confirmando as observações realizadas por outros autores como Couto et al. (1995), no Saco do Limoeiro, Paraná; Oliveira \& Mochel (1999), em um manguezal impactado do Maranhão, e Franklin-Junior (2000), em áreas estuarinas da Paraíba. Os resultados de matéria orgânica encontrados no rio Pacoti são semelhantes aos resultados registrados por Bezerra (2005), também realizados neste estuário.

Mochel (1995) apontou a matéria orgânica como fator determinante na disponibilidade de alimento para os organismos do ecossistema e heterogeneidade do habitat. Fundos com teores mais elevados de silte e argila, geralmente com maior teor de matéria orgânica, tiveram maior abundância de comedores de depósito, pois essas feições favorecerem a forma de alimentação dos organismos (Bemvenuti, 1992).

Em relação à salinidade, o banco areno-lamoso estudado foi caracterizado como ambiente eurihalino, tendo apresentado durante a maré baixa uma variação de 36 a 40,5 ao longo dos pontos amostrados. De acordo com vários autores (Oliveira et al. 1988; Mochel, 1995; Irving, 1991; Rosa-Filho, 1997; Oliveira \& Mochel, 1999; Ysebaert et al., 2003), a salinidade é um parâmetro essencial na caracterização da comunidade bêntica, sendo comum encontrar, segundo Rosa-Filho (1997), um gradiente de ocorrência de espécies tipicamente 
marinhas em função deste parâmetro. Este efeito da salinidade demonstrado por estes autores, em geral, se estabelece horizontalmente ao longo do rio, o que corresponde às variações de meso-escala propostas por Zajac (1999). Em uma escala menor, como no banco areno-lamoso estudado, as variações de salinidade parecem influenciar mais na abundância que na composição e distribuição dos organismos (Miranda et al., 1988), refletindo um efeito temporal de salinidade na sobrevivência dos organismos durante a maré baixa.

O oxigênio dissolvido da água de percolação do rio Pacoti apresentou valores bastante baixos ao longo do banco em condições de baixamar. O teor de oxigênio dissolvido presente em bancos lamosos é altamente influenciado pelo hidrodinamismo, as características granulométricas do sedimento e a topografia do local, que interagem, permitindo uma maior ou menor oxidação do sedimento (Franklin-Júnior, 2000). Segundo Mattisson \& Lindén (1983), em sedimentos com elevado conteúdo orgânico, a atividade microbiana é intensificada, apresentando como consequência o aumento no consumo de oxigênio. Segundo Oliveira et al. (1988), o zoobentos foi considerado altamente adaptado à instabilidade do sedimento, ao alto teor de matéria orgânica associada e ao consequente baixo teor de oxigênio dissolvido. Porém, Irving (1991) afirmou que em condições de baixas concentrações de oxigênio, ocorre redução na riqueza específica e dominância da fauna por poliquetas de corpo mole. Em uma avaliação em pequena escala, as variações no teor de oxigênio refletem as condições hidrodinâmicas locais, as quais determinam não somente a distribuição dos sedimentos finos e do detrito, mas também na oxigenação da camada mais superficial do substrato.

Tanto o grau de umidade do sedimento como a dessecação, fatores relacionados com a temperatura, são de extrema importância na distribuição de indivíduos que vivem em regiões entremarés. O banco areno-lamoso estudado caracterizou-se pela presença de uma fina cobertura irregular de algas filamentosas, o que proporcionou maior retenção de água e consequente favorecimento do habitat aos organismos. Áreas com substrato abrigado, ainda que parcialmente, possuem maior estabilidade ambiental, proporcionando condições mais favoráveis para o estabelecimento e manutenção das populações bentônicas (Wijnsma et al., 1998). Este fato pode ter contribuído para as elevadas densidades de indivíduos, principalmente poliquetas, ao longo dos pontos amostrados.

Segundo Oliveira et al. (1988), nas regiões com sedimentos mistos ou lodosos, caracterizados por alto teor de matéria orgânica, normalmente encontram-se crustáceos tanaidáceos e moluscos telinídeos. Apesar dos moluscos telinídeos não estarem presentes, os tanaidáceos da família Apseudidae ocorreram em altas densidades, estabelecendo agregados principalmente nos pontos onde a proporção de areia fina foi mais elevada.

As relações entre a distribuição dos bentos e as variáveis sedimentológicas têm sido discutidas por muitos autores (Reise, 1985; McCall \& Tevesz, 1982; Ishikawa, 1989; Alongi \& Christoffersen, 1992). Vários autores têm registrado que sedimentos com proporções elevadas de silte e argila, como aqueles encontrados em planícies flúvio-marinhas de ambientes estuarinos, são propícios para a vida de organismos bentônicos, visto que tais sedimentos: apresentam maiores proporções de matéria orgânica, que podem servir como principal fonte de alimento para muitas espécies; possuem alta abundância de microrganismos, dada a maior razão superfície/área de partículas finas; estão associados a ambientes de baixa hidrodinâmica, favorecendo a fixação e a locomoção dos organismos epifaunais e cavadores superficiais, e o assentamento das larvas planctônicas; e são tipicamente moles, facilitando a locomoção e os movimentos escavatórios da infauna cavadora (Reise, 1985; Silva-Camacho et al., 2016). 
No presente estudo, duas condições distintas dos sedimentos definiram a distribuição dos táxons mais abundantes. A alta densidade de poliqueta tubícola Laeonereis culveri (10.422 ind./ $\mathrm{m}^{2}$ ) em áreas com maior porcentagem de areia, fator que contribuiu para maior movimento de água e consequentemente maior concentração de oxigênio no sedimento; e áreas com porcentagens mais equilibradas de finos e areia favoreceram às altas densidades dos tanaidáceos Apseudidae (1641 ind./ m²), que atuam na desarticulação dos grãos sedimentares para a remoção de partículas orgânicas. Oliveira et al. (1988) também observaram esta estreita relação entre a ocorrência de crustáceos tanaidáceos e moluscos marinhos com sedimentos mistos ou lamacentos com alto teor de matéria orgânica nas planícies estuarinas do rio Pacoti, no Ceará.

$\mathrm{Na}$ área estudada, foi evidenciada diferença nos valores de $\mathrm{CaCO}_{3}$ e cascalho no sedimento. Estes parâmetros estão fortemente correlacionados, uma vez que a maior parte do cascalho observado constituiu-se de fragmentos de conchas e de restos de troncos da vegetação do manguezal. No estuário do rio Pacoti, Oliveira et al. (1988) encontraram conchas vazias e fragmentadas de moluscos na margem do banco de areia que emerge durante a maré baixa, porém no presente trabalho pode-se observar que restos de troncos foram os maiores responsáveis pela composição do cascalho encontrado nesta área, em especial nas subáreas 6 e 7, na margem interna do banco (em direção ao rio).

A macroinfauna no banco areno-lamoso estudado caracterizou-se pela predominância de poliquetas, moluscos e crustáceos, com percentuais de $88 \%, 11 \%$ e $1 \%$ respectivamente. Oliveira et al. (1988) já haviam destacado no estuário do rio Pacoti, a ocorrência destes três grupos taxonômicos. Em estudos sobre endofauna de planícies lamosas entremarés, Oliveira \& Mochel (1999) registraram a mesma taxocenose, porém com valores de dominância de 72\% de poliquetas, $15 \%$ de crustáceos e $8 \%$ moluscos bivalves. A dominância dos poliquetas em áreas estuarinas é fato comum na maior parte dos trabalhos de caracterização de macrofauna bentônica (Couto et al., 1995; Dittman, 2000; Franklin-Junior, 2000; Faraco \& Lana, 2003; French et al.; 2004; Sampaio, 2004). Sampaio (2004) comparou a macrofauna em bosques de mangues do Pará, norte do Brasil, e observou uma dominância dos poliquetas com $72 \%$ do total dos invertebrados coletados. Ele afirmou que a maior participação desse grupo em áreas de manguezais parece estar relacionada às condições locais de mobilidade e alimentação.

Dentre os poliquetas, as espécies Laeonereis culveri e Capitella spp. foram as mais representativas do grupo no banco areno-lamoso do rio Pacoti, contabilizando respectivamente $68 \%$ e $14 \%$ do total de organismos da macrofauna. Estas espécies são comumente encontradas com larga escala em áreas estuarinas (Netto \& Lana, 1994).

Segundo Klesh (1970), L. culveri é uma espécie que possui um importante papel na teia trófica de áreas estuarinas altamente produtivas. No presente trabalho, esta foi a mais representativa da poliquetofauna contabilizando $77 \%$ do total das espécies de poliquetas e $68 \%$ do total de invertebrados da macroinfauna. Isso pode estar relacionado às condições que essa espécie tem em responder ao estresse ambiental provocado pelas baixas concentrações de oxigênio dissolvido, elevadas taxas de temperatura e salinidade encontradas na área estudada. Pinto \& Bemvenuti (2003), pesquisando as perturbações biológicas e antrópicas sobre as associações de meio e macrofauna em regiões estuarinas, observou que em áreas próximas a efluentes aumentava significativamente a dominância de poliquetas da espécie Laeonereis acuta (= L. culveri), organismos menos seletivos, os quais se comportam como oportunistas, em consequência do enriquecimento orgânico.

As espécies da família Capitellidae estão entre os poliquetas mais comumente encontrados em sedimentos inconsolidados e são tolerantes a uma larga taxa de variação de 
temperatura e salinidade (Blake, 2000). Segundo Irving (1991), uma redução ocasional da tensão de oxigênio dissolvido estimula o incremento populacional das espécies oportunistas como, Capitella capitata, em detrimento de espécies menos resistentes. No presente estudo, esta espécie foi encontrada em altas densidades em regiões pobres em oxigênio, ocorrendo como segunda espécie mais abundante no banco areno-lamoso do rio Pacoti. Nos ambientes estuarinos, Capitella capitata foi uma das primeiras espécies designadas como "indicador de poluição", devido a sua dominância em ambientes de sedimentos ricos em matéria orgânica e com altas taxas de distúrbios ou estresse (Blake, 2000).

No banco areno-lamoso do estuário do rio Pacoti, os resultados de abundância foram bem elevados, possivelmente devido às altas densidades dos poliquetas, principalmente da espécie Laeonereis culveri encontrada nesta área. A grande dominância de poliquetas refletiu na redução dos valores de equitabilidade e, por conseguinte, da diversidade. Este resultado concorda com Couto et al. (1995), que destacaram que os valores de diversidade são tipicamente afetados pelos padrões de dominância numérica da fauna local e apenas secundariamente pela riqueza de espécies. Alguns autores sugerem que a diversidade das espécies diminui quando os organismos são expostos a vários estresses físicos, ou seja, características definidas como baixa diversidade em ambientes tropicais entremarés, estão relacionadas ao estresse natural ao qual a zona está submetida (Alongi, 1989; Martin et al., 1993).

De acordo com Rosa-Filho (1997), um grande número de fatores tem sido citado como controladores da distribuição espacial e temporal da abundância e diversidade dos organismos bentônicos em estuários. Os valores máximos dos índices de diversidade no banco areno-lamoso estudados $(0,7$ a 1,3) foram mais elevados que os encontrados por Oliveira \& Mochel (1999) em estuários do Maranhão, inferiores aos de Couto et al. (1995) no Saco do Limoeiro, Paraná e semelhantes ao trabalho de Wijnsma et al. (1998) em Mauritânia, na África. Porém, Mochel (1995), estudando a endofauna do manguezal de Coroa Grande, no Rio de Janeiro, considerou índices de diversidade altos, aqueles com valores próximos a 3,5.

O banco areno-lamoso do rio Pacoti apresentou alta similaridade faunística entre as subáreas. Embora na área tenham sido reconhecidos dois grupos distintos, estes apresentaram alto grau de semelhança, refletindo um banco areno-lamoso bastante homogêneo, apesar de sua heterogeneidade ambiental. Em uma análise em pequena escala, verificou-se que a espécies se distribuíram em função das características do sedimento (a seleção do grão e os percentuais de carbonato de cálcio e de matéria orgânica), definindo assim a formação de agregações (manchas), ainda que pouco discriminadas, das espécies mais abundantes sobre o banco.

Ao longo da zona estuarina, as condições do ambiente bentônico variam as escalas de heterogeneidade do habitat de acordo com as forçantes locais (por exemplo, variação de maré, vazão do rio, circulação estuarina). Segundo diversos autores, esta variação resulta de diferentes combinações de fatores físicos e biológicos agindo sobre escalas espaciais e temporais variáveis (por exemplo, Zajac 1999; Zajac et al., 2000; Knebel \& Poppe, 2000). Assim, no presente estudo, onde um único banco areno-lamoso do estuário do rio Pacoti foi avaliado, as características espaciais em pequena escala representaram condições distintas que influenciaram na distribuição da macroinfauna, reforçando a íntima relação dos organismos bentônicos com os sedimentos que habitam e descrevendo um padrão de distribuição na área que, em avaliações horizontais de meso-escala, não seriam percebidas. 
Agradecimentos - À Fundação Cearense de Apoio ao Desenvolvimento Científico e Tecnológico (FUNCAP) pelo financiamento do projeto, ao prof. Dr. Paulo da Cunha Lana e profa Dra. Cinthya Simone Gomes Santos do Centro de Estudos do mar (CEM) da Universidade Federal do Paraná, pelo estágio concedido à primeira autora e à MSc Rossana Cristina de Azevedo pelo apoio na identificação de poliquetas.

\section{REFERÊNCIAS BIBLIOGRÁFICAS}

Alongi, D.M. \& Christoffersen, P. Benthic infauna and organism-sediment relations in a shallow, tropical coastal area: influence of outwelles mangrove detritus and physical disturbance. Mar. Ecol. Prog. Series, v. 81, p. 229-245, 1992.

Alongi, D.M. Ecology of tropical soft-bottom benthos: a review with emphasis on energy concepts. Rev. Biol. Trop., v. 37, n.1, p. 85-100, 1989.

Bemvenuti, C.E. Interações Biológicas da Macrofauna Bentônica numa enseada estuarina da Lagoa dos Patos, RS, Brasil. Tese de doutorado, Instituto Oceanográfico da Universidade de São Paulo, 1992.

Bezerra, L.E.A. Distribuição espacial e Aspectos Populacionais de caranguejos do gênero Uca Leach, 1814 (Crustacea: Decapoda) no Manguezal do Rio Pacoti (Aquiraz-CE). Dissertação de mestrado em Ciências Marinhas Tropicais, 2005.

Blake, J.A. Family Capitellidae Grube, 1862. In: Blake, J.A.; Hilbig, B. \& Scott, P.H. (eds.). The Annelida, Part 4. Polychaeta (Flabelligeridae to Ampharetidae). Taxonomic Atlas of the benthic fauna of the Santa Maria Basin and Western Santa Bárbara Channel, vol. 7. Santa Bartbara Museum of Natural History, Santa Barbara California, 2000.

Caswell, H. \& Cohen, J.E. Communities in patchy environments: a model of disturbance, competition, and heterogeneity. In: Kolasa J. \& Pickett S.T. (eds) Ecological Heterogeneity, New York, NY: Springer-Verlag, p 97-122, 1991.

Clarke, K.R. \& Warwick, R.M. Change in Marine Communities: An Approach to Statistical Analysis and Interpretation. Phymouth Marine Laboratory, p. 91, 1994.

Couto, E.C.G.; Almeida, M.V.O. \& Lana, P.C. Diversidade e distribuição da macrofauna bêntica do Saco do Limoeiro Ilha do Mel, Paraná - Outono de 1990. Publção esp. Inst. Oceanogr., São Paulo, v. 11, p. 239-247, 1995

Dittman, S. 2000. Zonation of benthic communities in a tropical tidal flat of northeast Australia. Zentrum für Marine Tropenökologie, Bremem.

Faraco, L.F.D. \& Lana, P.C. Response of polychaetes to oil spills in natural and defaunated subtropical mangrove sediments from Paranaguá bay (SE Brazil). Hydrobiologia, v. 496, p. 321-328, 2003.

Franklin-Júnior, W. Macrofauna Bentônica da Região entremarés de bancos areno-lamosos em um estuário Tropical: Rio Mamanguape, Paraíba, Brasil. Dissertação (Mestrado do Centro de Ciências exatas e da Natureza) - Universidade Federal da Paraíba, João Pessoa, 2000.

French, K.; Robertson, S. \& O`Donnell, M.A. Differences in invertebrate infaunal assemblages of constructed and natural tidal flats in New South Wales, Australia. Estuar. Coast. Shelf. Sci., p. 173-183, 2004. 
Irving, M.A. 1991. Estrutura da Macrofauna bêntica da Zona Entremarés de Serpetiba (Rio de Janeiro - Brasil): Aspectos Descritivos e Metodológicos. Tese apresentada ao Instituto Oceanográfico da Universidade de São Paulo, para obtenção do título de doutor em ciências. 178p, São Paulo.

Ishikawa, K. 1989. Relationship between bottom characteristics and benthic organisms in the shallow water of Oppa Bay, Miyagi. Mar. Biol., v. 102, p. 265-273.

Kjerfve, B.; Lacerda, L.D. \& Diop, H.S. 1997. Mangrove Ecosystem Studies in Latin America and Africa. United Nations Educational, 349p. Paris.

Klesh, W.L. 1970. The reproductive biology and larval development of Laeonereis culveri Wesbster (Polychaeta; Nereididae). Contrib. Mar. Sci., v. 15.

Knebel, H.J. \& Poppe, L.J. Sea-floor environments within Long Island Sound: a regional overview. J. Coast. Res., p. 533-550, 2000.

Lacerda L.D. \& Sena, D.D. Estimativa de cargas der Nitrogênio, Fósforo e metais pesado de Interesse ambiental para as bacias inferiores do litoral do estado do Ceará. Zoneamento Ecológico econômico (ZEE) da Zona Costeira do Estado do Ceará Fortaleza, 2005.

Lana, P.C.M; Couto, E.C.G. \& Almeida, M.V.O. Polychaete distribution and abundance in intertidal flats of Paranaguá Bay (SE Brazil). Bull. Mar. Sci., v. 60, n. 2, p. 433-442, 1997.

Martin, D.; Ballesteros, E.G.J.M. \& Palacín, C. Small-scale Structure of infaunal Polychaete Communities in an Estuarine Environment: Methodological Approach. Estuar. Coast. Shelf. Sci., v. 36, p. 47-58, 1993.

Mattisson, J. \& Linden, O. Benthic macrofauna succession under mussels, Mytilus edulis L. (Bivalvia, cultures on hanging long-lines. Sarsia, v. 68, p.97-102, 1983.

McCall, P.L. \& Tevesz, M.J.S. Animal -Sediment Relations. The Biogenic Alteration of Sediments. v. 2, 336p, 1982.

Miranda, P.T.C.; Gurgel, F.F.G.; Liberato, M.A.F.; Oliveira, M.T. \& Arruda, T.L.B. Comunidades bentônicas em raízes de Rhizophora mangle Linnaeus, no manguezal do rio Ceará (Ceará-Brasil). Arq. Ciên. Mar, v. 27, p. 101-110, Fortaleza, 1988.

Mochel, F.R. Endofauna do manguezal. São Luís: Editora da Universidade Federal do Maranhão. 120p, 1995.

Monteiro, U.H.L. Estudos das áreas de mangue entre os estados Piauí e Pernambuco com um enfoque para o estado do Ceará nos anos de 1978 e 1999/2004 utilizando sensoriamento remoto. Dissertação de mestrado em Ciências Marinhas tropicais da Universidade Federal do Ceará - Instituto de Ciências do Mar, 99p. Fortaleza, 2005.

Morisita, M. I o-Index, a measure of dispersion of individuals. Res. Popul. Ecol., v. 4, n. 1, p. 1-7, 1962.

Morrisey, D.J.; Howitt, L.; Underwood, A. J. \& Stark, J. S. Spatial Variation in soft-sediment benthos. Mar. Ecol. Prog. Ser., v. 81 p. 197-204. 1992.

Nanami, A.; Saito, H.; Akita, H.; Motomatsu, K \& Kuwahara, H. Spatial distribution and assemblage structure of macrobenthic invertebrates in a brackish variables. Estuar. Coast. Shelf. Sci., v. 63, p. 167-176, 2005. 
Netto, S.A. \& Lana, P.C. Effects of sediment disturbance on the structure of benthic fauna in a subtropical tidal creek of southeastern Brazil. Mar. Ecol. Prog. Ser., v. 106, p. 239-247, 1994.

Oliveira, A.M.E.; Irving, M.A. \& Lima, H.H. Aspectos bioecológicos do estuário do Rio Pacoti, Ceará, Brasil. Arq. Ciên. Mar, v. 27, p. 91-100, 1988.

Oliveira, V.M. \& Mochel, F.R. Macroendofauna bêntica de substrato móveis de um manguezal sob impacto das atividades humanas no sudoeste da Ilha de São Luís, Maranhão, Brasil. Depto de Oceanografia e Limnologia. Bol. Lab. Hidrobiol., v. 12, p. 75-93, 1999.

Pinheiro, D.R. Análise do comportamento hidrodinâmico e sedimentológico do Estuário do Rio Piranji-CE (NE/BRASIL). Dissertação de Mestrado em Geociências. Universidade Federal do Rio Grande do Sul. Programa de Pós-graduação em geociências, Porto Alegre, 2003.

Pinto, T.K.D.O. \& Bemvenuti, C.E. Effects of burrowing macrofauna on meiofauna community structure. Acta Limnol. Bras., v.15, n.3, p. 41-51, 2003.

Reise, K. Tidal Flat Ecology. An Experimental Approach to Species Interactions. Springer-Verlag. 191 p. 1985.

Rosa-Filho, J.S. Caracterização das Associações de Macroinvertebrados de Fundos Moles dos Estuários do Rio Grande do Sul (Brasil). Situação de verão. Dissertação de mestrado em Oceanografia Biológica da Universidade de Rio Grande, 1997.

Sampaio, D.S. Comparação da macrofauna bentônica em bosques de mangue sob diferentes graus de degradação no Município de Bragança, Pará, Brasil. Dissertação de mestrado em Ecologia de Ecossistemas costeiros e estuarinos. Universidade Federal do Pará, Bragança, 84p., 2004.

Schaffner, L.C. Small-scale organism distributions and patterns of species diversity: Evidence for positive interactions in an estuarine benthic community. Mar. Ecol. Prog. Ser., Oldendorf, v. 61, n. 1, p. 107-117, 1990.

Silva-Camacho, D.D.S.; Gomes, R.D.S.; Santos, J.N. \& Araújo, F.G. Distribution of benthic fauna in sediment grains and prop roots of a mangrove channel in south-eastern Brazil. Jour. Mar. Biol. Assoc.U.K., p. 1-9, 2016.

Wijnsma, G.; Wolff, W. J.; Meijboom, A.; Duiven, P. \& Vlas, J.D. Species richness and distribuition of benthic tidal flat fauna of the Banc d'Arguin, Mauritania. Oceanologica ACTA, v. 22, 1998.

Ysebaert, T.; Herman, P.M.J.; Meire, P.; Craeymeersch. J.; Verbeek, H. \& Heip, C.H.R. Large-scale spatial patterns in estuaries: estuarine macrobenthic communities in the Schelde estuary, NW Europe. Estuar. Coast. Shelf. Sci., v. 57, p. 335-355, 2003.

Zajac, R.N. Understanding the seafloor landscape in relation to assessing and managing impacts on coastal environments. pp 211-227. In: J.S. Gray, W. Ambrose Jr., A. Szaniawska (eds) Biogeochemical Cycling and Sediment Ecology, Kluwer Publishing, Dordrecht, 1999.

Zajac, R.N., Lewis, R.S., Poppe, L.J., Twichell, D.C., Vozarik, J. \& DiGiacomo-Cohen, M.L. Relationships among sea-floor structure and benthic communities in Long Island Sound at regional and benthoscape scales. J. Coast. Res., 627-640, 2000. 